\title{
Respiratory viral infections post-lung transplantation
}

\author{
Katherine M. Vandervest • Martin R. Zamora
}

Published online: 20 June 2012

(C) Springer Science+Business Media, LLC 2012

\begin{abstract}
Community-acquired respiratory viruses (CARVs) are common pathogens in lung transplant recipients. Infection due to these viruses is associated with multiple complications including: rhinitis, pharyngitis, bronchitis, pneumonia, respiratory failure and even death. CARVs have also become increasingly recognized as a risk factor for acute rejection (AR) and bronchiolitis obliterans syndrome (BOS). Newer diagnostic techniques have enhanced the accuracy of diagnosis, but proven treatment options for CARVs are limited. Further insight into the immune response and allograft dysfunction associated with CARV infections is needed in order to develop novel management strategies which can reduce the morbidity and mortality caused by these infectious agents.
\end{abstract}

Keywords Lung transplantation · Immunocompromised ·

Rhinovirus · Adenovirus · Influenza $\cdot$ Parainfluenza .

Respiratory syncytial virus · Human metapneumovirus ·

Ribavirin - Acute rejection - Bronchiolitis obliterans

syndrome $\cdot$ PCR

\section{Introduction}

Despite important advances in the field of lung transplantation over the last few decades, survival for lung transplant recipients remains significantly lower than other solid organ transplants (SOT). This outcome is believed to be due in

K. M. Vandervest $(\square) \cdot$ M. R. Zamora

Division of Pulmonary Sciences and Critical Care Medicine,

University of Colorado Hospital,

Anschutz Medical Campus, 1635 Aurora Ct, Suite 7020,

Mail Stop F-749,

Aurora, CO 80045, USA

e-mail: katherine.vandervest@ucdenver.edu part to exposure of the allograft to the external environment. Inhalation of environmental agents such as infectious organisms can be a trigger for immunologically mediated, deleterious effects on lung function. The community-acquired respiratory viruses (CARVs) such as respiratory syncytial virus (RSV), parainfluenza virus (PIV), influenza A and B (Flu), adenovirus (ADV), human metapneumovirus (hMPV), coronavirus, and rhinovirus (RV) are increasingly recognized as important pathogens in lung transplant recipients [1-5].

Studies suggest that CARVs may have immediate and long-term adverse effects on allograft performance $[1-4,6 \bullet$, 7-9]. Viral infections generate direct cytopathic damage to lung epithelium and provoke indirect injury through the stimulation of inflammatory cytokines and $\mathrm{T}$ cell activation $[6 \bullet]$. This alloreactive response to viral infection can lead to substantial morbidity and mortality in lung transplant recipients. The development of potent antiviral agents, sensitive molecular techniques for the detection of infection, and comprehensive management strategies is paramount to reducing complications from CARVs following lung transplantation. This review discusses the individual pathogens, the incidence of disease, the role of new diagnostic techniques, treatment regimens for established viral infections, prevention strategies, and the potential impact of CARVs on acute and chronic allograft rejection.

\section{Community-acquired respiratory viruses (CARVs)}

Epidemiology

The CARVs are a diverse group of viruses belonging to several families including the Paramyxoviridae (RSV, PIV, hMPV), the Orthomyxoviridae (FLU A and B), the Picornaviridae (RV), Coronaviridae (Coronavirus), and the 
Adenoviridae (ADV). They are all single-stranded RNA viruses except for adenovirus which is a double-stranded, DNA virus. Viral transmission follows direct inoculation of infected secretions from fomites or by large-particle aerosols into the upper respiratory tract. Rhinovirus and PIV typically occur year-round while the remaining CARVs usually have seasonal peaks between October and April [4, 6•].

The reported incidence of CARVs in lung transplant patients ranges from $2-21 \%$ [4, 9-12]. Their actual incidence, however, may be underestimated due to insensitive diagnostic methods and under reporting by lung transplant recipients. Lung allografts may be particularly susceptible to viral infections due to immunosuppression, cough inhibition from denervation of the transplanted lung, impaired mucociliary clearance, and compromised lymphatic drainage [3, 4]. Multiple studies demonstrate no difference in CARV infection rates based on demographics or maintenance immunosuppressant regimens [6•, 9].

The time between transplant and infection is variable and can develop within days to years post-transplantation. One single-center, retrospective study indicated that $24 \%$ of diagnosed PIV infections developed within 90 days of lung transplantation [11]. The early onset of CARV illness suggests that donor organ transmission is conceivable [5].

\section{Clinical syndromes}

Similar to immunocompetent hosts, CARV infections can cause a spectrum of disease processes including: rhinitis, pharyngitis, tracheobronchitis, bronchiolitis, and pneumonia. Progression to a lower respiratory tract infection (LRTI) has been reported to occur in as few as $6 \%$ of infected recipients [6•] and as many as $66 \%$ [2]. Most studies define LRTI by a variable combination of the following signs and symptoms: infiltrate on thoracic imaging, positive bronchoalveolar lavage (BAL) specimen for virus, decrease in $\mathrm{FEV}_{1}$ by $>10 \%-15 \%$, hypoxemia, and wheezing $[6 \cdot 12]$. Kumar et al. reported that outcomes of asymptomatic versus symptomatic patients were not significantly different [6•]. Those patients who are symptomatic most commonly exhibit cough, fever, nasal congestion, wheezing, hypoxemia, coryza, and dyspnea. Mortality in lung transplant recipients following CARV infections ranges from 3-20\% [11-13].

Chest radiographs typically have nonspecific findings including: no change from baseline, diffuse interstitial infiltrates, or focal alveolar consolidation. Chest CT scans of lung transplant recipients infected with RSV reveal evidence of airways disease including air trapping, ground-glass infiltrates, air-space consolidation, bronchial dilation, bronchial wall thickening, and bronchiolitis [14, 15]. Adenoviral infection may be more frequently associated with progressive pulmonary opacities [15]. Serial CT scans can show persistent bronchial thickening, air-trapping, and "mosaic" lung attenuation which are manifestations of bronchiolitis obliterans syndrome (BOS) or chronic rejection.

CARV infections may adversely affect pulmonary physiology or $\mathrm{FEV}_{1}$. In two studies, RSV caused a significant decrease in $\mathrm{FEV}_{1}(>10 \%-20 \%)$ within ninety days of infection $[11,13]$. A more recent prospective analysis, inclusive of all the recognized CARVs, revealed that a fall in $\mathrm{FEV}_{1}$ of $>20 \%$ ensued in one-third of lung transplant recipients and that this fall in $\mathrm{FEV}_{1}$ was persistent in the majority of patients supporting an association of CARVs with BOS $\left[6^{\bullet}\right]$.

\section{Diagnosis}

Prompt, accurate diagnosis of CARV infection is critical for the ongoing management of lung transplant recipients given the concern for associated acute and chronic rejection. Standards for diagnostic tests include the immunofluorescent antibody assay (DFA) and respiratory culture performed on nasal wash, nasal swab, or BAL specimens $[2,6 \bullet, 16]$. Compared to pediatric patients, the yield obtained from upper respiratory tract samples in adults may be inferior due to decreased viral burden or shedding in the nasal passages [2,11]. Analysis by BAL has been shown to improve the sensitivity of results [2]. Viral culture can also take many days for a positive result to return which delays initiation of antiviral therapy and risks transmission of illness to other immunosuppressed patients. These older forms of testing typically identify fewer numbers of the CARVs. Newer tests such as rapid respiratory viral culture (RRV) and antigen detection (EIA) allow for faster and more precise results [17]. At the present time, however, no commercially available antigen detection kits exist for rhinovirus and coronavirus.

Multiplex, polymerase chain reaction (PCR) analysis is rapidly becoming the preferred test for viral diagnostics in immunocompromised hosts. This diagnostic test allows for the detection of up to 19 common respiratory viral types with a single assay [6•]. Weinberg et al. [17] found that PCR improved the sensitivity of viral detection to $84 \%$ versus $67 \%$ for RRV culture and $54 \%$ for EIA. Kumar et al. [6] also demonstrated higher sensitivity for PCR (98\%) compared to DFA and viral culture (69\%). One drawback of PCR is that it cannot discern between viable and killed virus during the course of antiviral therapy [18] so clinicians must rely on RRV or traditional viral culture to determine treatment efficacy.

Treatment

\section{Influenza virus (FLU)}

The influenza viruses are the only CARVs for which specific FDA-approved therapy exists. Influenza is typically 
caused by influenza A or B virus. Annual pandemics of FLU subtypes such as H1N1, H5N1, H2N3 have been noted to cause varying degrees of graft dysfunction in lung transplant recipients. A review of the 2009 Australian H1N1 pandemic in lung transplant recipients revealed an overall incidence of $3 \%$ (24 patients), with allograft dysfunction in $75 \%$ and death in $21 \%$ [19]. Current antiviral therapy for FLU is directed against proteins contained in the viral envelope. The amantanes or M2 inhibitors consisting of amantadine and rimantidine, block ion channels within the membrane. Unfortunately, efficacy for this class of drugs is limited due to the rapid development of antiviral resistance and the lack of effect against FLU-B [3, 20].

The neuraminidase inhibitors, oseltamavir (Tamiflu), zanamivir (Relenza), and peramivir, inhibit the release of new virus from the host cell which prevents the spread of the virus. According to Centers for Disease Control recommendations, zanamivir should be reserved for oseltamivirresistant strains of FLU [21]. The inhaled form of this medication should be used with caution in patients with serious preexisting respiratory disease due to the potential side effects of cough, bronchospasm, and even death. If inhaled zanamivir is contraindicated, then an intravenous form has been approved for use in emergency situations. Intravenous peramivir is also only approved under an emergency use authorization. Similar to the M2 inhibitors, immunocompromised patients have been shown to have higher resistance to neuraminidase inhibitors possibly due to prolonged viral shedding leading to selection of resistant strains [21]. In general, early initiation of antiviral therapy for influenza reduces the risk of developing viral pneumonia ( $0 \%$ versus $25 \%)$, and of death $(0 \%$ versus $25 \%$ ) [22•].

Investigational therapies for FLU are in development, but there has been no report of use in immunosuppressed patients. Favipiravir (T705, Toyama Chemical) and DAS181 (Fludase, NexBio Inc.) have demonstrated in vitro and in vivo effectiveness against FLU including H1N1 and avian strains of H5N1. There is also published data for the use of RNA-interference-based (RNAi) antiviral agents [23]. Preventative treatment of FLU with inactivated or killed viral vaccinations is an option for transplant recipients, but the efficacy of vaccines is likely decreased due to immune suppression [24]. In a study by Issa et al. only $51 \%$ of patients had protective antibody titers of 1:40 or higher [25]. The live, attenuated, nasally administered vaccination, Flumist, is contraindicated in transplant patients due to risk of infection.

\section{Parainfluenza virus (PIV)}

While there are currently no FDA-approved antiviral medications or vaccines for the treatment of PIV, ribavirin has been shown to have both in vitro and in vivo activity against PIV [21]. A case report of its use in combination with IVIG and methylprednisolone revealed a slower decline in $\mathrm{FEV}_{1}$ for lung and heart-lung transplant recipients when compared to placebo [26]. DAS181 (Fludase, NexBio Inc.), the investigational drug for FLU, inhibits PIV infection in vitro and in animal models. There has additionally been a case report of its successful use in inhaled form for a lung transplant recipient with a proven PIV infection [27]. This agent disables airway epithelial receptors by removal of sialic acid moieties thereby preventing viral attachment [27]. Antiviral RNAi-based therapeutic targets for PIV are also under investigation [23]. A live attenuated vaccine against PIV-3 is in development for preventative therapy. However, the live variant of virus in the inoculation renders it unsafe for immunosuppressed patients who could acquire a serious infection from vaccination.

\section{Respiratory syncytial virus (RSV)}

Aerosolized ribavirin is licensed for the treatment of RSV bronchiolitis in infants and children. Use of the humanized monoclonal antibody, palivizumab (Synagis), is also associated with a $55 \%$ decrease in hospitalization of pediatric patients [13]. Although there is limited data for treating RSV following lung transplantation [13] many experts recommend administration of inhaled ribavirin, IVIG, methylprednisolone, and palivizumab $[4,11,13,25]$. Vilchez et al. reported a decrease in the incidence of post-RSV BOS to $15 \%$ with the use of inhaled ribavirin [3] compared to an incidence of $32 \%$ in those without therapy [1]. Another study in hematopoietic stem cell transplant patients with RSV pneumonia revealed a mortality of $31 \%$ with combination therapy (IVIG, steroids, ribavirin, and palivizumab) versus $100 \%$ in those who were untreated or experienced a delay in treatment [5]. The exact mechanism of action of ribavirin is unclear. The drug is a synthetic nucleoside analog which may interfere with the expression of viral mRNA and proteins at the translatory level. It may also be incorporated into the RSV RNA viral genome and lead to "lethal mutagenesis" [13]. Immunoglobulin therapy neutralizes RSV antibody titers and palivizumab is directed against the fusion protein of RSV.

The use of alternate routes of ribavirin administration which may decrease respiratory side effects, minimize toxic exposure to healthcare workers, and reduce treatment costs have been examined. A prospective analysis by Glanville et al. suggested that intravenous ribavirin therapy with oral steroids in lung transplant recipients is safe and cost effective [13]. Two other studies found that use of oral ribavirin either prevented post-RSV BOS up to 1.5 years after treatment [12] or significantly improved graft function recovery after paramyxoviral infection [28]. 
Investigational medications for RSV treatment are also in development. The RNAi drug ALN-RSV01 was investigated by Zamora et al. in a randomized controlled trial of twenty-four lung transplant recipients. The inhaled medication resulted in lower symptom scores and significantly lower rates of new onset BOS at ninety days when compared to placebo [29•]. Anti-viral isoprenylation inhibitors like lovastatin could also be efficacious in RSV treatment by inhibiting viral replication and cell to cell fusion [30].

\section{Human metapneumovirus virus (hMPV)}

Human metapneumovirus was recognized as a CARV in 2001. The incidence of hMPV infection in lung transplant patients has been reported to range from $6 \%$ [31] to $31 \%$ $[32,33]$. Multiple studies have described an association of hMPV infection to acute rejection (AR) with incidences up to $63 \%$ [32]. As a more recently recognized CARV, treatment options for hMPV are less well studied. No specific therapy for hMPV exists, but ribavirin has been shown to have in vitro activity against the virus consistent with other members of the Paramyxoviridae family. Published case reports indicate successful treatment of hMPV with intravenous ribavirin monotherapy or in conjunction with IVIG and steroids in lung transplant recipients [34]. Novel research evaluating the possibility of synthetic, interference RNAs (RNAi) as therapeutic agents against hMPV demonstrated complete inhibition of viral replication in vitro and partial inhibition in a murine model [35].

\section{Adenovirus (ADV)}

No definitive therapy exists for ADV. Traditional therapy has included supportive care with decreased immunosuppression. A role for antiviral agents remains unproven, but several case reports have described in vitro activity against ADV with ribavirin. Our center has utilized inhaled ribavirin with IVIG in three patients with ADV pneumonia. Two patients improved after five days of treatment and the third patient with preexisting BOS developed respiratory failure and died (nonpublished data). More recently, the use of cidofovir for the treatment of adenoviral disease has been described to reduce mortality in pediatric lung transplant and adult hematopoietic stem cell transplant recipients [36, 37]. A lipid conjugate of cidofovir, CMX001, has also been evaluated in case series of immunocompromised patients with limited success [38]. This agent has the benefit of less nephrotoxicity then cidofovir, but may have the side effect of diarrhea.

\section{Rhinovirus $(R V)$}

No proven antiviral therapy is available for the treatment of $\mathrm{RV}$ which is perhaps the most common cause of CARV infection in healthy and immunocompromised adults. Pleconaril which binds to the rhinoviral capsid and prevents viral uncoating or attachment leading to decreased replication has been studied as a potential pharmacotherapeutic option. Investigational trials in immunocompetent adults revealed a shorter time to alleviation of illness and decreased frequency of positive cultures from nasal secretions with treatment [39]. Reports of utilization of pleconaril in lung transplant recipients with rhinoviral pneumonia are limited and proof of its efficacy is still lacking [40]. There is additional concern that a substantial number of clinical isolates have limited sensitivity to pleconaril or exhibit high effective inhibitory concentrations [40].

\section{Relationship of CARVs to acute and chronic rejection}

Many studies have supported an association between CARVs and AR $[2,6 \cdot, 7,11,13,41,42]$. CARVs may play an important role in AR by upregulation of cytokine production and activation of $\mathrm{T}$ cells resulting in an inflammatory allopathic response within the graft. In a prospective analysis by Kumar et al., the primary endpoint of a decrease in $\mathrm{FEV}_{1}$ by at least $20 \%$ or evidence of at least $\mathrm{A} 2$ rejection on biopsy occurred in $33 \%$ of lung transplant recipients versus $6.5 \%$ in recipients without a CARV infection [6 ]. Another report found that $79 \%$ of lung transplant recipients diagnosed with a CARV infection had a fall in $\mathrm{FEV}_{1}$ of greater than $10 \%$ [11] and a third study indicated that $82 \%$ of patients with a transbronchial biopsy at the time of infection had evidence of acute allograft rejection [2]. In some of these studies, however, the $\mathrm{FEV}_{1}$ returned to baseline within weeks to months [11].

Thus the association of CARVs with a persistent decline in $\mathrm{FEV}_{1}$ or BOS is unclear. In murine orthotopic lung transplant models, infection with PIV type 1 resulted in persistent epithelial injury, luminal obliteration, and chronic bronchiolar scarring [43, 44]. Several human investigations, however, have yielded conflicting results regarding the risk of developing BOS after CARV infection. These studies have varied in the number of viruses researched, the sample sizes, the sensitivity of methodologies used, and the frequency of sample collection, making it difficult to draw specific conclusions.

A single-center, retrospective study involving predominantly upper-respiratory tract infections (URTIs), did not find a connection between RSV or PIV and BOS although both viruses contributed to long-term dysfunction or death in $33 \%$ of patients [11]. Another review of multiple cohorts found a pooled incidence of $18 \%$ of BOS in virus positive cases versus $11.6 \%$ in virus negative cases which was not statistically significant [45]. Several other retrospective studies, however, have supported an association between 
CARVs and chronic rejection [1-3, 7, 8, 11, 33, 41, 46]. A review of cohort studies by Vilchez et al. in 2003 reported that the rate of BOS following CARV infection in lung transplant recipients ranges from $32-50 \%$ depending on the type of virus [3]. Billings et al. suggested that recipients with LRTIs due to a CARV had a relative risk of 2.3 for developing high-grade BOS and that recipients infected within the first six months post-transplant were more likely to acquire high-grade BOS [1]. Further support for an independent connection between CARV infection and BOS was published in a 2004, retrospective study by Khalifah et al. [8]. This analysis indicated that CARV infection after lung transplantation doubled the risk of developing BOS stage 1 and if it was a LRTI the risk of subsequent progression to BOS was tripled.

Current prospective analyses also provide inconsistent data for the relationship between CARV infection and BOS in lung transplant recipients. A multicenter study by Milstone et al., which predominantly followed patients with URTIs for one year post respiratory infection, did not find a difference in the rates of BOS development between infected and non-infected recipients with RSV or PIV [9]. In contrast, a single-center investigation by Gottlieb et al. with $40.8 \%$ of patients exhibiting LRTI-symptoms, indicated that symptomatic CARV infections increased the probability for new onset of BOS, but not progression. Risk was particularly increased following PIV infection [47•]. A third prospective analysis which evaluated up to nineteen CARVs over a three-year period post-infection reported a $62 \%$ incidence of BOS I at one year [6•].

\section{Conclusions}

Lung transplant recipients are frequently exposed to CARVs. These viruses are becoming increasingly recognized as an important cause of morbidity and mortality in lung transplant recipients. Both upper and lower respiratory tract infections have been shown to occur with all of the CARVs and newer, highly sensitive diagnostic techniques have led to improvements in the diagnostic accuracy of results. Although studies evaluating the impact of CARVs on short and long term allograft function are heterogenous and have various limitations, the majority of the investigations support a direct relationship between AR and CARV infection. This also supports an association between CARV infection and BOS since AR is an established risk factor for BOS. At the present time, proven treatment for CARV infection in lung transplant recipients is extremely limited. Combination therapy with ribavirin has been reported to have activity against RSV, PIV, hMPV, and ADV. Alternative medications for the treatment of Paramyxoviridae infection as well as FLU, ADV, and RV are in development.
Further insight into disease pathogenesis of CARV infections through future prospective studies is warranted in order to hasten the implementation of novel management strategies and decrease the risk of BOS which is the major cause of long-term graft failure in lung transplant recipients.

Disclosure K. M. Vandervest: none; M. R. Zamora: Alnylam Pharmaceuticals (grant).

\section{References}

Papers of particular interest have been highlighted as: - Of importance

1. Billings JL, Marshall HI, Savik K, Wendt CH. Respiratory viruses and chronic rejection in lung transplant recipients. J Heart Lung Transplant. 2002;21:559-66.

2. Vilchez RA, Dauber J, McCurry K, et al. Parainfluenza virus infection in adult lung transplant recipients: an emergent clinical syndrome with implications on allograft function. Am J Transplant. 2002;3:116-20.

3. Vilchez RA, Dauber J, Kusne S. Infectious etiology of bronchiolitis obliterans: the respiratory viruses connection - myth or reality? Am J Transplant. 2003;3:245-9.

4. Wendt $\mathrm{CH}$. Community respiratory viruses: organ transplant recipients. Am J Med. 1996;102:31-6.

5. Zamora MR. Viral infections complicating lung and solid organ transplantation. In: Lynch JP, Ross DJ, editors. Lung and heartlung transplantation. New York: Taylor \& Francis Group; 2006. p. $485-514$.

6. - Kumar D, Husain S, Hong Chen M, et al. A prospective molecular surveillance study evaluating the clinical impact of community-acquired respiratory viruses in lung transplant recipients. Transplantation. 2010;89:1028-33. A prospective survey of CARVS and their impact in lung transplant recipients.

7. Kumar D, Erdman D, Keshavjee S, et al. Clinical impact of community-acquired respiratory viruses on bronchiolitis obliterans after lung transplant. Am J Transplant. 2005;5:2031-6.

8. Khalifah AP, Hachem RR, Chakinala MM, et al. Respiratory viral infections are a distinct risk for bronchiolitis obliterans syndrome and death. Am J Respir Crit Care Med. 2004;170:181-7.

9. Milstone AP, Brumble LM, Barnes J, et al. A single-season prospective study of respiratory viral infections in lung transplant recipients. Eur Respir J. 2006;28:131-7.

10. Gerna G, Vitulo P, Rovida F, et al. Impact of human metapneumovirus and human cytomegalovirus versus other respiratory viruses on the lower respiratory tract infections of lung transplant recipients. J Med Virol. 2006;78:408-16.

11. McCurdy LH, Milstone A, Dummer S. Clinical features and outcomes of paramyxoviral infection in lung transplant recipients treated with ribavirin. J Heart Lung Transplant. 2003;22:745-53.

12. Pelaez AG, Marshall L, Force SD, et al. Efficacy of oral ribavirin in lung transplant patients with respiratory syncytial virus lower respiratory tract infection. J Heart Lung Transplant. 2009;28:67-71.

13. Glanville AR, Scott AI, Morton JM, et al. Intravenous ribavirin is a safe and cost-effective treatment for respiratory syncytial virus infection after lung transplantation. J Heart Lung Transplant. $2005 ; 24: 2114-9$.

14. Ko JP, Shepard JA, Sproule MW, et al. CT manifestations of respiratory syncytial virus infection in lung transplant recipients. J Comput Assist Tomogr. 2000;24:735-42. 
15. Matar LD, McAdams HP, Palmer SM, et al. Respiratory viral infections in lung transplant recipients: radiologic findings with clinical correlation. Radiology. 1999;213:735-42.

16. Whimbey E, Englund JA, Couch RB. Community respiratory virus infections in immunocompromised patients with cancer. Am J Med. 1997;102:10-8.

17. Weinberg A, Zamora MR, Li S, et al. The value of polymerase chain reaction for the diagnosis of viral respiratory tract infections in lung transplant recipients. J Clin Virol. 2002;25:171-5.

18. Englund JA, Whimbey EE. Community acquired respiratory viruses after hemopoietic stem cell or solid organ transplantation. In: Bowden RA, Ljungman P, Paya CV, editors. Transplant infections. 2nd ed. Philadelphia: Lippincott-Williams and Wilkens; 2003. p. 375-98.

19. Ng BJ, Glanville AR, Snell G, et al. The impact of pandemic influenza A H1N1 2009 on Australian lung transplant recipients. Am J Transplant. 2011;11:568-74.

20. Englund JA. Antiviral therapy for influenza. Semin Pediatr Infect Dis. 2002; 13:120-8.

21. Moss RB, Steigbigel RT, Sanders RL, Fang F. Perspective: emerging challenges in the treatment of influenza and parainfluenza in transplant patients. Adv Virol. 2011;2011:1-8.

22. - Ison MG. Antiviral therapies for respiratory viral infections in lung transplant patients. Antivir Ther. 2012;17:193-200. A recent review on the epidemiology and management strategies for CARVS in lung transplant patients.

23. DeVincenzo JP. The promise, pitfalls and progress of RNAinterference-based antiviral therapy for respiratory viruses. Antivir Ther. 2012, In press.

24. Ison MG, Hayden FG. Viral infections in immunocompromised patients: what's new with respiratory viruses? Curr Opin Infect Dis. 2002;15:355-67.

25. Issa NC, Marty FM, Gagne LS, et al. Seroprotective titers against 2009 H1N1 influenza a virus after vaccination in allogeneic hematopoietic stem cell transplantation recipients. Biol Blood Marrow Transplant. 2010;17:434-8.

26. Liu V, Dhillon GS, Weill D. A multi-drug regimen for respiratory syncytial virus and parainfluenza virus infections in adult lung and heart-lung transplant recipients. Transpl Infect Dis. 2010;12:38-44.

27. Guzman-Suarez BB, Buckley MW, Gilmore ET, et al. Clinical potential of DAS181 for treatment of parainfluenza-3 infections in transplant recipients. Transpl Infect Dis. 2012;0:1-7.

28. Fuehner T, Dierich M, Duesberg C, et al. Single-centre experience with oral ribavirin in lung transplant recipients with paramyxovirus infections. Antivir Ther. 2011;16:733-40.

29. - Zamora MR, Budev M, Rolfe M, et al. RNA interference therapy in lung transplant patients infected with respiratory syncytial virus. Am J Respir Crit Care Med. 2011;183:531-8. Use of a novel technology, inhaled siRNA, for treatment of RSV in lung transplant recipients.

30. Gower TL, Graham BS. Antiviral activity of lovastatin against respiratory syncytial virus in vivo and in vitro. Antimicrob Agents Chemother. 2001;45:1231-7.

31. Weinberg A, Lyu DM, Li S, et al. Incidence and morbidity of human metapneumovirus and other community-acquired respiratory viruses in lung transplant recipients. Transpl Infect Dis. 2010;12:330-5.
32. Hopkins P, McNeil K, Kermeen F, et al. Human metapneumovirus in lung transplant recipients and comparison to respiratory syncytial virus. Am J Respir Crit Care Med. 2008;178:876-81.

33. Larcher C, Geltner C, Fischer H. Human metapneumovirus infection in lung transplant recipients: clinical presentation and epidemiology. J Heart Lung Transplant. 2005;24:1891-901.

34. Raza K, Ismailjee SB, Crespo M, et al. Successful outcome of human metapneumovirus (hMPV) pneumonia in a lung transplant recipient treated with intravenous ribavirin. J Heart Lung Transplant. 2007;26:862-4.

35. Darniot M, Schildgen V, Schildgen O, et al. RNA interference in vitro and in vivo using DsiRNA targeting the nucleocapsid $\mathrm{N}$ mRNA of human metapneumovirus. Antiviral Res. 2012;93:36473.

36. Doan ML, Mallory GB, Kaplan SL, et al. Treatment of adenovirus pneumonia with cidofovir in pediatric lung transplant recipients. J Heart Lung Transplant. 2007;26:883-9.

37. Neofytos D, Ojha A, Mookerjee B, et al. Treatment of adenovirus disease in stem cell transplant recipients with cidofovir. Biol Blood Marrow Transplant. 2007;13:74-81.

38. Florescu DF, Pergam SA, Neely MN, et al. Safety and efficacy of CMX001 as salvage therapy for severe adenovirus infections in immunocompromised patients. Biol Blood Marrow Transplant. 2012;18:731-8.

39. Hayden FG, Herrington DT, Coats TL, et al. Pleconaril Respiratory Infection Study Group. Efficacy and safety of oral pleconaril fortreatment of colds due to picornaviruses in adults: results of 2 double-blind, randomized, placebo-controlled trials. Clin Infect Dis. 2003;36:1523-32.

40. Kaiser L, Aubert JD, Pache JC, et al. Chronic rhinoviral infection in lung transplant recipients. Am J Respir Crit Care Med. 2006;174:1392-9.

41. Garantziotis S, Howell DN, McAdams HP, et al. Influenza pneumonia in lung transplant recipients: clinical features and association with bronchiolitis obliterans syndrome. Chest. 2001;119:1277.

42. Husain S, Singh N. Bronchiolitis obliterans and lung transplantation: evidence for an infectious etiology. Semin Respir Infect. 2002;17:310.

43. Winter JB, Gouw AS, Groen M, Wildevuur C, Prop J. Respiratory viral infections aggravate airway damage caused by chronic rejection in rat lung allografts. Transplantation. 1994;57:418-22.

44. Winter JB, Groen M, Welling S, van der Logt K, Wildevuur CR, Prop J. Inadequate antibody response against respiratory viral infection in long-surviving rat lung allografts. Transplantation. 1995;59:1583-9.

45. Vu DL, Bridevaux PO, Aubert JD, et al. Respiratory viruses in lung transplant recipients: a critical review and pooled analysis of clinical studies. Am J Transplant. 2011;11:1071-8.

46. Bridges ND, Spray TL, Collins MH, Bowles NE, Towbin JA. Adenovirus infection in the lung results in graft failure after lung transplantation. J Thorac Cardiovasc Surg. 1998;116:617-23.

47. - Gottlieb J, Schulz TF, Welte T, et al. Community-acquired respiratory viral infections in lung transplant recipients: a single season cohort study. Transplantation. 2009;87:1530-7. A recent update on the potential role of CARVs in acute and chronic lung allograft rejection. 